\title{
Diabetic phenotype and prognosis of patients with heart failure and preserved ejection fraction in a real life cohort
}

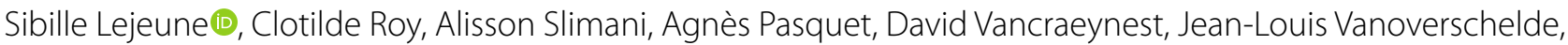
Bernhard L. Gerber, Christophe Beauloye ${ }^{\dagger}$ and Anne-Catherine Pouleur ${ }^{*}+$

\begin{abstract}
Background: Heart failure with preserved ejection fraction (HFpEF) is a heterogeneous syndrome, with several underlying etiologic and pathophysiologic factors. The presence of diabetes might identify an important phenotype, with implications for therapeutic strategies. While diabetes is associated with worse prognosis in HFpEF, the prognostic impact of glycemic control is yet unknown. Hence, we investigated phenotypic differences between diabetic and non-diabetic HFpEF patients (pts), and the prognostic impact of glycated hemoglobin (HbA1C).
\end{abstract}

Methods: We prospectively enrolled 183 pts with HFpEF (78 \pm 9 years, 38\% men), including 70 (38\%) diabetics (type 2 diabetes only). They underwent 2D echocardiography $(n=183)$, cardiac magnetic resonance (CMR) $(n=150)$, and were followed for a combined outcome of all-cause mortality and first HF hospitalization. The prognostic impact of diabetes and glycemic control were determined with Cox proportional hazard models, and illustrated by adjusted Kaplan Meier curves.

Results: Diabetic HFpEF pts were younger ( $76 \pm 9$ vs $80 \pm 8$ years, $p=0.002$ ), more obese (BMl $31 \pm 6$ vs $27 \pm 6 \mathrm{~kg} /$ $\left.\mathrm{m}^{2}, \mathrm{p}=0.001\right)$ and suffered more frequently from sleep apnea ( $\left.18 \% \mathrm{vs} 7 \%, \mathrm{p}=0.032\right)$. Atrial fibrillation, however, was more frequent in non-diabetic pts (69\% vs $53 \%, p=0.028)$. Although no echocardiographic difference could be detected, CMR analysis revealed a trend towards higher LV mass ( $66 \pm 18 \mathrm{vs} 71 \pm 14 \mathrm{~g} / \mathrm{m}^{2}, \mathrm{p}=0.07$ ) and higher levels of fibrosis ( $53 \%$ vs $36 \%$ of patients had ECV by T1 mapping $>33 \%, \mathrm{p}=0.05$ ) in diabetic patients.

Over $25 \pm 12$ months, $111 \mathrm{HFpEF}$ pts (63\%) reached the combined outcome (24 deaths and $87 \mathrm{HF}$ hospitalizations). Diabetes was a significant predictor of mortality and hospitalization for heart failure (HR: 1.72 [1.1-2.6], $p=0.011$, adjusted for age, BMI, NYHA class and renal function). In diabetic patients, lower levels of glycated hemoglobin (HbA1C < 7\%) were associated with worse prognosis (HR: 2.07 [1.1-4.0], $p=0.028$ adjusted for age, BMI, hemoglobin and NT-proBNP levels).

Conclusion: Our study highlights phenotypic features characterizing diabetic patients with HFpEF. Notably, they are younger and more obese than their non-diabetic counterpart, but suffer less from atrial fibrillation. Although diabetes

\footnotetext{
*Correspondence: anne-catherine.pouleur@uclouvain.be

${ }^{\dagger}$ Christophe Beauloye, Anne-Catherine Pouleur contributed equally Division of Cardiology, Department of Cardiovascular Diseases, Cliniques Universitaires St. Luc and Pôle de Recherche Cardiovasculaire (CARD), Institut de Recherche Expérimentale et Clinique (IREC), Cardiovascular Division, Université Catholique de Louvain, Avenue Hippocrate, 10, 1200 Brussels, Belgium
}

(c) The Author(s) 2021. This article is licensed under a Creative Commons Attribution 4.0 International License, which permits use, sharing, adaptation, distribution and reproduction in any medium or format, as long as you give appropriate credit to the original author(s) and the source, provide a link to the Creative Commons licence, and indicate if changes were made. The images or other third party material in this article are included in the article's Creative Commons licence, unless indicated otherwise in a credit line to the material. If material is not included in the article's Creative Commons licence and your intended use is not permitted by statutory regulation or exceeds the permitted use, you will need to obtain permission directly from the copyright holder. To view a copy of this licence, visit http://creativeco mmons.org/licenses/by/4.0/. The Creative Commons Public Domain Dedication waiver (http://creativecommons.org/publicdomain/ zero/1.0/) applies to the data made available in this article, unless otherwise stated in a credit line to the data. 
is a predictor of poor outcome in $\mathrm{HFpEF}$, intensive glycemic control $(\mathrm{HbA1C}<7 \%)$ in diabetic patients is associated with worse prognosis.

Keywords: Heart failure and preserved ejection fraction, Type 2 diabetes, HbA1C, Prognosis

\section{Background}

Heart failure with preserved ejection fraction (HFpEF) is increasingly being recognized as an umbrella term describing a heterogeneous group of clinical and pathophysiological phenotypes. HFpEF is a diagnostic challenge, especially since important features are mainly apparent on exercise and require dynamic testing [1]. Furthermore, the phenotypic heterogeneity among patients is a key reason for current lack of treatment improving outcome. Indeed most recent clinical trial using sacubitril-valsartan in HFpEF had disappointing results [2], although it could decrease the rate of hospitalisation in specific subgroups [3]. All eyes are now turned towards ongoing studies with sodium-glucose cotransporter-2 inhibitors (SGLT-2i) [4]. Type 2 diabetes (T2D) is one of the most frequent comorbidity associated with HFpEF (prevalence varying from 33 to $43 \%$ ) [5], but there are still numerous uncertainties surrounding the mechanisms by which these two conditions interact. There is a need to understand the clinical characteristics of patients with HFpEF and diabetes in order to guide therapeutic decision making, highlight potential phenotype-specific targets, and aid in the development of risk stratification tools. Sub studies of large clinical trials (RELAX-HF [6], I-PRESERVE [7], CHARM [8] and TOPCAT [9]) comparing diabetic and nondiabetic patients showed that HFpEF patients with T2D were younger, more obese, displayed greater structural echocardiographic abnormalities (higher left ventricular mass) and had a worse prognosis than patients without T2D. Those studies were clinical trials with restrictive inclusion criteria and might not reflect HFpEF patients encountered in daily practice. The same differences in clinical characteristics were found in a large American registry (GWTG-HF registry) [10] but imaging parameters were not available for analyzes. Previous studies $[11,12]$ showed a U-shaped association between $\mathrm{HbA1C}$ and prognosis in heart failure patients. Those studies either were conducted among patients with HFrEF alone, or did not make a distinction between patients according to ejection fraction. Glycemic variability was found to be associated with diastolic dysfunction and with poor outcome in HFpEF [13, 14], but data remain limited. Accordingly, we aimed to investigate phenotypic differences between diabetic and nondiabetic patients with $\mathrm{HFpEF}$ in a prospective, real life cohort. The prognostic impact of glycemic control assessed by $\mathrm{HbA} 1 \mathrm{C}$ was also evaluated in this population.

\section{Methods}

\section{Study population}

Patients with HFpEF encountered in our division of cardiology between December 2015 and June 2017 (in hospital and at ambulatory visits) were prospectively screened for inclusion in the study. Inclusion and exclusion criteria were reported in previous publications [15]. Briefly, the following criteria had to be fulfilled: New York Heart Association (NYHA) functional class $\geq$ II, typical signs of HF, NT-proBNP $>350 \mathrm{pg} / \mathrm{ml}$ and/or an hospitalization for HF in the previous 12 months, left ventricular ejection fraction $\geq 50 \%$, and relevant structural heart disease (left ventricular (LV) hypertrophy/ left atrial (LA) enlargement) and/or diastolic dysfunction by echocardiography [16]. The exclusion criteria were: history of reduced ejection fraction $(\mathrm{LVEF}<50 \%)$, severe valvular disease, infiltrative or hypertrophic cardiomyopathy, acute coronary syndrome in the previous 30 days, severe chronic obstructive pulmonary disease, congenital heart disease, pericardial disease, atrial fibrillation (AF) with a ventricular response $>140 \mathrm{bpm}$, and severe anemia (hemoglobin $<8 \mathrm{~g} / \mathrm{dl}$ ). A total of 183 patients satisfied the inclusion criteria. Patients underwent blood sampling and complete transthoracic echocardiography and cardiac magnetic resonance (CMR) in the absence of following contra-indications: pacemaker, claustrophobia or estimated glomerular filtration rate $(\mathrm{eGFR})<30 \mathrm{~mL} / \mathrm{min} / 1.73 \mathrm{~m}^{2}(\mathrm{~N}=151)$. The local ethics committee approved the study, and all patients gave written informed consent before study enrolment (Clinical trial NCT03197350). The investigation conforms to the principles outlined in Declaration of Helsinki.

\section{Clinical data}

Patients were interrogated about symptoms, medical history and treatment and were thoroughly examined. Other information, including diagnosis and treatment of diabetes were retrieved from medical files and from review of hospital records.

\section{Echocardiography}

Standardized complete transthoracic echocardiography (TTE) exams were acquired according to established guidelines using iE33 ultrasound systems (Philips Medical Systems, Andover, Massachusetts) equipped with a 
3.5/1.75-MHz phased-array transducer and stored on a XCELERA 2.1 PACS server (Philips Medical Systems, Andover, Massachusetts).

\section{Cardiac magnetic resonance}

CMR was performed using a $3 \mathrm{~T}$ system (Ingenia, Philips Medical Systems, Best, The Netherlands). The different sequences have been previously described [17]. Pre- and post-contrast MOLLI images were processed using the open-source software MRmap v1.4 under IDL. Pre- and post-myocardial $\mathrm{T} 1$ times were measured in six regions of interest in the myocardium (anterior, anterolateral, inferolateral, inferior, inferoseptal, anteroseptal). We calculated the average T1 time of the six different regions of interest. Areas of ischemic focal fibrosis identified by late gadolinium enhancement (LGE) were excluded from the analysis. Extracellular volume (ECV) was then computed according to the formula [18]. A cut off of ECV $>33 \%$ was used to define significant diffuse myocardial fibrosis [17].

\section{Follow up}

Patients were prospectively followed by ambulatory visits and phone calls at 6-months intervals. Clinical and survival status was obtained by follow up visits and by phone contact with the patients, their relatives, or their physician if necessary. The primary endpoint was a composite of all-cause mortality or hospitalization for HF, whichever came first. Hospitalization was defined as patients diagnosed with heart failure and requiring intravenous diuretics, either treated in the emergency room or admitted to the hospital.

\section{Statistical analysis}

Statistical analyses were performed using SPSS version 25 (SPSS Corp., Somers, New York). All tests were 2-sided and $\mathrm{p}$-value $<0.05$ was considered statistically significant. Continuous variables were expressed as mean \pm 1 standard deviation (SD) and categorical variables as count and proportion. Differences of characteristics between groups were examined using independent sample t-test or Chi square test when appropriate. Uni- and multivariate Cox regression analyzes were used to determine the prognostic impact of diabetes and HbA1C. Diabetic patients enrolled in the study who completed the follow up $(67 / 70,96 \%)$ and with at least one HbA1c measurement in the three months previous to inclusion were used for analyzes about the prognostic impact of glycemic control (62/70, 89\%) (Additional file 1). Adjusted Kaplan Meier curves were used to illustrate event-free survival of HFpEF patients. The log-rank test was used to compare survival among different groups.

\section{Results \\ Characteristics and outcome of diabetic versus nondiabetic HFpEF patients (Table 1)}

The total population was constituted of $183 \mathrm{HFpEF}$ patients (78 \pm 9 years, $62 \%$ women), including 70 (38\%) diabetics. Diabetic HFpEF patients were younger ( $76 \pm 9$ vs $80 \pm 8$ years, $\mathrm{p}=0.002$ ) and more obese (body mass index (BMI) $31 \pm 6$ vs $27 \pm 6 \mathrm{~kg} / \mathrm{m}^{2}, \mathrm{p}=0.001$ ). They suffered more frequently from chronic coronary artery disease $(47 \%$ vs $24 \%, \mathrm{p}=0.001)$ and obstructive sleep apnea ( $18 \%$ vs $7 \%, p=0.032)$. Atrial fibrillation, however, was more frequent in nondiabetic patients ( $69 \%$ vs $53 \%, p=0.028)$. Although no echocardiographic difference could be detected between the two groups, CMR analysis revealed a trend towards higher LV mass in the diabetic population $\left(66 \pm 18\right.$ vs $71 \pm 14 \mathrm{~g} / \mathrm{m}^{2}$, $\mathrm{p}=0.07)$. Interestingly, more diabetic patients $(53 \% \mathrm{vs}$ $36 \%, \mathrm{p}=0.05$ ) had high levels of myocardial fibrosis (defined as ECV by T1 mapping > 33\%) [17]. The main differences between diabetic and nondiabetic patients are summarized in Fig. 1.

The follow up was completed for 177 (97\%) patients, including 67 diabetics (96\%) over a mean duration of $30 \pm 9$ months. Over this period of time, 27/67 (40\%) diabetic patients died, and 52/67 (78\%) reached the combined outcome, versus 28/110 (25\%) deaths and $59 / 110$ (54\%) combined outcome in the nondiabetic group. As such, T2D was associated with worse prognosis in univariate Cox regression (HR 1.65 [1.1-2.4], $\mathrm{p}=0.009$ ). Although it shortly missed statistical significance for mortality alone, the association between diabetes and single outcomes taken separately went in the same direction (for all-cause mortality HR 1.58 [0.92.7], $\mathrm{p}=0.092$ and for hospitalization HR 1.64 [1.12.5 ], $\mathrm{p}=0.022$ ). After adjustment for age, body mass index, NYHA functional class and glomerular filtration rate, diabetes remained a significant predictor of mortality and hospitalization for heart failure (HR: 1.72 [1.1-2.6], $\mathrm{p}=0.011$ ) as shown by the adjusted Kaplan Meier curves (Fig. 2). 
Table 1 Clinical, echocardiographic and CMR characteristics of diabetic versus nondiabetic HFpEF patients

\begin{tabular}{|c|c|c|c|}
\hline & Nondiabetic $\mathrm{N}=113(62 \%)$ & Diabetic $N=70(38 \%)$ & p-value \\
\hline Age (years) & $80 \pm 8$ & $76 \pm 9$ & 0.002 \\
\hline Female (n, \%) & $71(63 \%)$ & $42(60 \%)$ & 0.70 \\
\hline Body mass index $\left(\mathrm{kg} / \mathrm{m}^{2}\right)$ & $27 \pm 6$ & $31 \pm 6$ & 0.001 \\
\hline NYHA III-IV (n, \%) & $60(53 \%)$ & $27(39 \%)$ & 0.056 \\
\hline Hospitalized for HF at inclusion $(n, \%)$ & $73(65 \%)$ & $43(61 \%)$ & 0.53 \\
\hline \multicolumn{4}{|l|}{ Atrial fibrillation (n, \%) } \\
\hline History & $78(69 \%)$ & $37(53 \%)$ & 0.028 \\
\hline At inclusion & $57(50 \%)$ & $26(37 \%)$ & 0.079 \\
\hline Ischemic cardiomyopathy (n, \%) & $27(24 \%)$ & $33(47 \%)$ & 0.001 \\
\hline Smoking (n, \%) & $50(45 \%)$ & $27(39 \%)$ & 0.42 \\
\hline Hypertension (n, \%) & $105(93 \%)$ & $67(97 \%)$ & 0.23 \\
\hline Hypercholesterolemia (n, \%) & $66(59 \%)$ & $49(70 \%)$ & 0.13 \\
\hline Sleep apneas (n, \%) & $8(7 \%)$ & $12(18 \%)$ & 0.032 \\
\hline $\operatorname{COPD}(n, \%)$ & $12(11 \%)$ & $7(10 \%)$ & 0.88 \\
\hline \multicolumn{4}{|l|}{ Medication } \\
\hline Loopdiuretics (n, \%) & $73(65 \%)$ & $51(73 \%)$ & 0.25 \\
\hline MRA $(n, \%)$ & $19(17 \%)$ & $13(19 \%)$ & 0.76 \\
\hline Beta blockers (n, \%) & $77(68 \%)$ & $41(59 \%)$ & 0.19 \\
\hline ACE inhibitors/ARB (n, \%) & $78(69 \%)$ & $46(66 \%)$ & 0.64 \\
\hline \multicolumn{4}{|l|}{ Biology } \\
\hline $\operatorname{eGFR}\left(\mathrm{ml} / \mathrm{min} / 1.73 \mathrm{~m}^{2}\right)$ & $58 \pm 22$ & $50 \pm 24$ & 0.026 \\
\hline Hemoglobin (g/dL) & $12 \pm 2$ & $11 \pm 2$ & 0.041 \\
\hline NT-proBNP (pg/mL) & 1937 [1040-3775] & $1745[955-3710]$ & 0.56 \\
\hline Troponin (pg/mL) & $22[13-37]$ & $31[17-42]$ & 0.034 \\
\hline \multicolumn{4}{|l|}{ Echocardiography } \\
\hline Indexed LA volume $\left(\mathrm{mL} / \mathrm{m}^{2}\right)$ & $46 \pm 19$ & $45 \pm 16$ & 0.67 \\
\hline LV ejection fraction (\%) & $62 \pm 7$ & $61 \pm 8$ & 0.35 \\
\hline E wave velocity (mm/s) & $91 \pm 32$ & $97 \pm 26$ & 0.23 \\
\hline Septal E/e' & $19 \pm 9$ & $20 \pm 7$ & 0.17 \\
\hline TAPSE (mm) & $19 \pm 5$ & $18 \pm 5$ & 0.40 \\
\hline eSPAP $(\mathrm{mmHg})$ & $43 \pm 11$ & $45 \pm 15$ & 0.27 \\
\hline CMR & $N=94$ & $N=57$ & \\
\hline CMR indexed LA Volume $\left(\mathrm{mL} / \mathrm{m}^{2}\right)$ & $70 \pm 31$ & $62 \pm 25$ & 0.12 \\
\hline CMR indexed LV EDV $\left(\mathrm{mL} / \mathrm{m}^{2}\right)$ & $72 \pm 18$ & $74 \pm 17$ & 0.37 \\
\hline CMR LV ejection fraction (\%) & $62 \pm 8$ & $62 \pm 9$ & 0.62 \\
\hline CMR indexed LV mass $\left(\mathrm{g} / \mathrm{m}^{2}\right)$ & $66 \pm 18$ & $71 \pm 14$ & 0.07 \\
\hline CMR RV ejection fraction (\%) & $56 \pm 8$ & $58 \pm 8$ & 0.41 \\
\hline CMR indexed RV EDV $\left(\mathrm{mL} / \mathrm{m}^{2}\right)$ & $79 \pm 25$ & $83 \pm 27$ & 0.36 \\
\hline$E C V>33 \%$ & $34(36 \%)$ & $28(53 \%)$ & 0.05 \\
\hline
\end{tabular}

Continuous variables are expressed as mean \pm 1 standard deviation (SD) and categorical variables as count and proportion. P-values are derived from independent sample t-test or Chi square test when appropriate

NYHA: New York Heart Association; COPD: chronic obstructive pulmonary disease; GFR: glomerular filtration rate estimated by CKD-epi; MRA: mineralocorticoid receptor antagonist; ACE: angiotensin-converting enzyme; ARB: angiotensin II receptor blockers LV: left ventricle; LA: left atrium; TAPSE: tricuspid annular plane systolic excursion; eSPAP: estimated systolic pulmonary artery pressures; CMR: cardiac magnetic resonance; EDV: end diastolic volume; RV: right ventricle; ECV: extracellular volume estimated by T1 mapping

\section{Characteristics and outcome of diabetic HFpEF patients according to glycemic control (Table 2)}

Overall, the diabetic patients in our population had well controlled diabetes with median HbA1C of 7.1 [6.1-7.8] \%. Almost half (32/65, 49\%) were treated with insulin, alone or in combination with Metformin. 

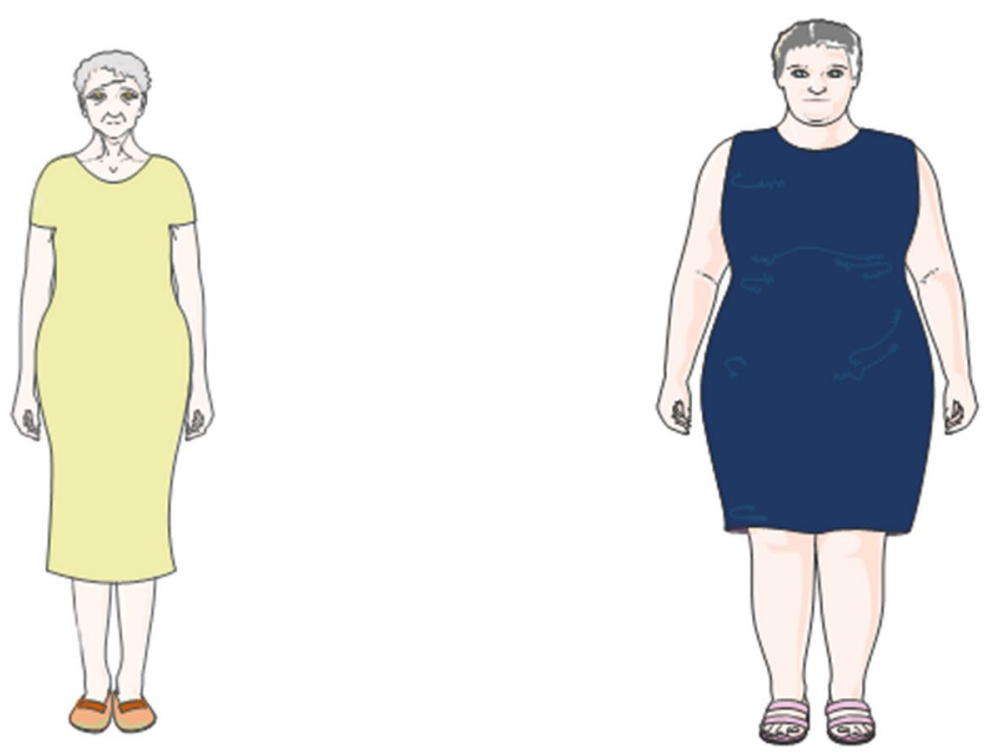

\begin{tabular}{|l|l|}
\hline \multicolumn{1}{|c|}{ Nondiabetic } & \multicolumn{1}{c|}{ Diabetic } \\
\hline Older & Younger \\
\hline Leaner & More obese \\
\hline More patients in NYHA class III - IV & $\begin{array}{l}\text { High prevalence of ischemic cardiomyopathy and } \\
\text { sleep apneas }\end{array}$ \\
\hline High prevalence of atrial fibrillation & Higher LV mass and more myocardial fibrosis \\
\hline
\end{tabular}

Fig. 1 Characteristics of diabetic versus nondiabetic HFpEF patients

Details of hypoglycemic treatments can be found in Fig. 3. Note that no patient was taking sodium-glucose cotransporter-2 inhibitors (SGLT-2i) as, in Belgium, they were reimbursed according to strict criteria at the time of inclusion. The subgroup of diabetic patients were compared among each other according to glycemic control (HbA1C $<7 \%$ versus $>7 \%$, Table 2 ). Patients with $\mathrm{HbA} 1 \mathrm{C}<7 \%$ were leaner, with a mean BMI of $29 \pm 6$ versus $32 \pm 7 \mathrm{~kg} / \mathrm{m}^{2}(\mathrm{p}=0.048)$. They had slightly lower hemoglobin levels and showed a tendency, although not statistically significant, toward higher NT-proBNP levels. The two groups were homogenous regarding age, sex and comorbidities, and had similar renal functions. Patients with $\mathrm{HbA1C}>7 \%$ were more often treated with insulin.

Out of 65 diabetic patients with HbA1C data, 62 (95\%) completed the follow up (Additional file 1). In 2 years, $15 / 31$ (48\%) diabetic patients with $\mathrm{HbA1C}<7 \%$ died and $28 / 31$ (90\%) reached the combined outcome, versus 10/31 (32\%) deaths in patients with $\mathrm{HbA1C}>7 \%$ and 20/31 (65\%) combined outcome. Lower levels of glycated hemoglobin were associated with worse prognosis (HR 2.07 [1.1-3.8], $\mathrm{p}=0.016$ ). Although it shortly missed statistical significance for hospitalization alone, the association between glycemic control and single outcomes taken separately went in the same direction (for all-cause mortality HR 2.36 [1.15.5], $\mathrm{p}=0.047$ and for hospitalization HR 1.86 [0.96-3.6], $\mathrm{p}=0.064$ ). After adjustment for age, body mass index, hemoglobin levels and NT-proBNP levels, HbA1C $<7 \%$ remained a significant predictor of mortality and hospitalization for heart failure (HR: 2.07 [1.1-4.0], $\mathrm{p}=0.028$ ). This can be seen in Fig. 4, showing the adjusted Kaplan Meier curves of event-free survival among diabetic HFpEF patients according to $\mathrm{HbA} 1 \mathrm{C}$ levels.

\section{Discussion}

The main findings of this study can be summarized as follows: 1. Diabetic patients with HFpEF show specific characteristics, including higher body mass index, lower prevalence of atrial fibrillation, lower hemoglobin levels and worse renal function. No echocardiographic difference could be detected, but CMR showed a trend towards higher LV mass and more myocardial fibrosis $(E C V>33 \%)$. 2. Diabetes is associated with an excess of adverse events (hospitalization for HF and mortality) in 


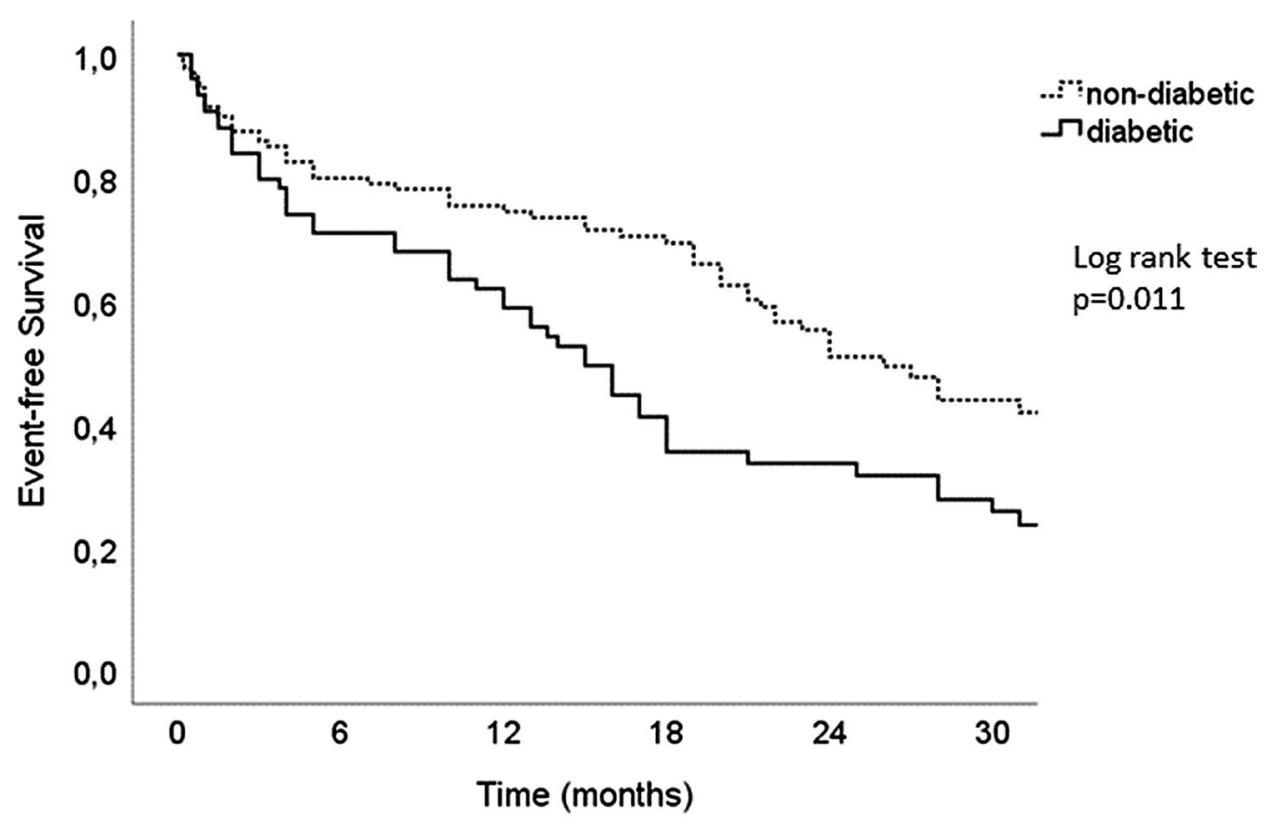

At risk:

$\begin{array}{rllllll}\text { Non diabetic } & 110 & 85 & 76 & 63 & 43 & 26 \\ \text { Diabetic } & 67 & 46 & 39 & 22 & 20 & 14\end{array}$

Fig. 2 Kaplan Meier curves of event-free survival in diabetic versus non-diabetic HFpEF patients. Adjustments were made for age, body mass index, NYHA functional class and glomerular filtration rate

HFpEF. 3. Lower levels of HbA1C levels are associated with worse prognosis in diabetic patients with HFpEF.

\section{Characteristics and outcome of diabetic versus nondiabetic HFpEF patients}

Regarding clinical characteristics, HFpEF patients with diabetes were younger and more obese than nondiabetic patients. This is consistent with sub studies from large clinical trials [6-8]. A large study examining age-related characteristics in HFpEF also observed that younger patients were more than twice as likely to be obese, and that the prevalence of diabetes ranged from $37 \%$ in the younger group versus $18 \%$ in the oldest group [19]. Although the reason for this difference is not completely elucidated, it might reflect that different pathophysiological pathways can lead to the development of HFpEF. The combination of diabetes and obesity, both conditions associated with a release of proinflammatory cytokines and decreased nitric oxide availability, could lead to the development of HFpEF at a younger age through myocardial remodelling and fibrosis [20]. Supporting this, diabetic patients also exhibited a trend towards higher LV masses and higher levels of myocardial fibrosis than their nondiabetic counterparts, consistently with previous studies
$[7,21,22]$. This can contribute to the worse prognosis conferred by diabetes, as we previously showed that extracellular matrix expansion (higher ECV by CMR) was associated with adverse events in HFpEF [17].

Atrial fibrillation, on the other hand, was more prevalent in the nondiabetic group. This is consistent with previously published literature [23-25]. AF and HFpEF often coexist and it is still unclear whether one affection leads sequentially to the other. More likely, the two disorders share a common mechanistic substrate, which causes AF and HFpEF [26, 27] and develop in parallel. A recent meta-analysis underlined that AF was associated with poor prognosis in $\mathrm{HFpEF}$, although it is unclear whether AF is only a marker of more severe heart failure, or a cause of mortality in itself [28]. Atrial fibrillation is also an age-related marker, hence, it is not surprising that the prevalence of AF is higher in the older nondiabetic group. Studies have also suggested differences in cardiac remodelling, with diabetic patients showing smaller LA volumes, that might contribute to this phenomenon [23]. However, the presence of AF was retrieved from medical files, patients interrogation, and a standard electrocardiogram at inclusion, but no long term rhythm monitoring was performed. As such, the prevalence of AF and other 
Table 2 Clinical characteristics of diabetic HFpEF patients according to glycemic control

\begin{tabular}{|c|c|c|c|c|}
\hline & Diabetic $N=65$ & $\mathrm{HbA} 1 \mathrm{C}<7 \% \mathrm{~N}=32$ & $\mathrm{HbA} 1 \mathrm{C}>7 \% \mathrm{~N}=33$ & p-value \\
\hline Age (years) & $76 \pm 9$ & $76 \pm 8$ & $75 \pm 10$ & 0.79 \\
\hline Duration of diabetes (years) & $19.3 \pm 8$ & $19.2 \pm 9$ & $19.4 \pm 8$ & 0.96 \\
\hline Female $(n, \%)$ & $42(60 \%)$ & $20(62 \%)$ & $20(61 \%)$ & 0.88 \\
\hline Body mass index $\left(\mathrm{kg} / \mathrm{m}^{2}\right)$ & $31 \pm 6$ & $29 \pm 6$ & $32 \pm 7$ & 0.048 \\
\hline NYHA III-IV (n, \%) & $27(39 \%)$ & $14(44 \%)$ & $12(36 \%)$ & 0.54 \\
\hline Hospitalized for HF at inclusion (n,\%) & $41(63 \%)$ & $21(66 \%)$ & $20(61 \%)$ & 0.55 \\
\hline Atrial fibrillation $(n, \%)$ & $37(53 \%)$ & $18(56 \%)$ & $16(48 \%)$ & 0.53 \\
\hline Ischemic cardiomyopathy $(\mathrm{n}, \%)$ & $33(47 \%)$ & $13(41 \%)$ & $18(55 \%)$ & 0.26 \\
\hline Smoking (n, \%) & 27 (39\%) & $10(31 \%)$ & $14(42 \%)$ & 0.35 \\
\hline Hypertension (n, \%) & $67(97 \%)$ & $32(100 \%)$ & $31(94 \%)$ & 0.49 \\
\hline Hypercholesterolemia (n, \%) & $49(70 \%)$ & $22(69 \%)$ & $24(73 \%)$ & 0.72 \\
\hline Sleep apneas (n, \%) & $12(18 \%)$ & $4(13 \%)$ & $8(27 \%)$ & 0.16 \\
\hline $\operatorname{COPD}(n, \%)$ & $7(10 \%)$ & $3(9 \%)$ & $3(9 \%)$ & 0.97 \\
\hline \multicolumn{5}{|l|}{ Biology } \\
\hline $\mathrm{HbA1C}(\%)$ & $7.1[6.1-7.8]$ & $6.1[5.8-6.5]$ & $7.7[7.2-8.4]$ & $<0.001$ by design \\
\hline $\operatorname{eGFR}\left(\mathrm{ml} / \mathrm{min} / 1.73 \mathrm{~m}^{2}\right)$ & $50 \pm 24$ & $49 \pm 27$ & $48 \pm 18$ & 0.78 \\
\hline Hemoglobin (g/dL) & $11 \pm 2$ & $11 \pm 2$ & $12 \pm 2$ & 0.046 \\
\hline NT-proBNP (pg/mL) & 1745 [955-3710] & 2373 [1148-5264] & 1464 [506-3696] & 0.086 \\
\hline \multicolumn{5}{|l|}{ Antidiabetic treatment } \\
\hline Insulin (n, \%) & $32(46 \%)$ & $11(34 \%)$ & $21(64 \%)$ & 0.018 \\
\hline Metformin $(n, \%)$ & 31 (44\%) & 15 (47\%) & 13 (39\%) & 0.54 \\
\hline Sulfonylureas (n, \%) & $16(23 \%)$ & $9(28 \%)$ & $5(15 \%)$ & 0.20 \\
\hline Gliptins (n, \%) & $8(11 \%)$ & $4(13 \%)$ & $4(12 \%)$ & 0.96 \\
\hline
\end{tabular}

Continuous variables are expressed as mean \pm 1 standard deviation (SD) and categorical variables as count and proportion. $p$-values are derived from independent sample t-test or Chi square test when appropriate

HbA1C: glycated hemoglobin; NYHA: New York Heart Association; COPD: chronic obstructive pulmonary disease; GFR: glomerular filtration rate estimated by CKD-epi

arrhythmias could have been underestimated in both groups.

The event rate in our study was high compared to clinical trials (16.1 / 100 persons-year overall mortality in the diabetic group versus $6.8-8.8$ in pooled data from I-Preserve, Charm-Preserved and TOPCAT [23]), but similar to a large community based study (15.2/100 persons-year [29]). Compared to clinical trials, our population is almost 10 years older ( 76 vs 69 years) had higher NT-proBNP levels (1745 vs 430-581 pg/mL), lower hemoglobin (11 vs $12.9-13.5 \mathrm{~g} / \mathrm{dL})$ and worse renal function ( 50 vs $62.7-71.4 \mathrm{~mL} / \mathrm{min} / 1.73 \mathrm{~m}^{2}$ ), all parameters associated with adverse events. The association between diabetic status and prognosis (hospitalization for HF and mortality) is consistent with the existing literature $[6,7,9$, 10, 23]. There are numerous pathophysiologic processes in diabetes that are thought to alter the myocardium resulting in less effective relaxation and contraction, including oxidative stress, inflammation and disorders in calcium transport, as well as alterations in substrate metabolism, and mitochondrial dysfunction [30, 31]. Furthermore, extra-cardiac effects of diabetes such as decreased arterial compliance, renal angiopathy, and autonomic dysfunction can also accelerate the progression of HFpEF [31]. In particular, hyperglycemia causes up-regulation of the sodium-glucose cotransporter-2 (SGLT-2) leading to increased proximal renal sodium absorption, volume expansion, and decreased responsiveness to diuretics [20,32, 33]. A better understanding of the interplay between diabetes and HF is crucial for the development of new therapies. This has recently been emphasized by the promising results of studies using SGLT-2 inhibitors in diabetic patients with HF [34, 35]. The results of ongoing randomized controlled trials using SGLT-2i in HFpEF $[4,20]$ are eagerly awaited. Nevertheless, a retrospective study showed less impressive effects of SGLT2i on cardiac remodeling in HFpEF compared to HFrEF, tempering enthusiasm for this class of treatment [35].

\section{Characteristics and outcome of diabetic HFpEF patients according to glycemic control.}

While the presence of diabetes conferred a worse prognosis to our HFpEF patients, tight glycemic control did not seem to reverse this association. On the contrary, patients with best controlled diabetes $(\mathrm{HbA} 1 \mathrm{C}<7 \%)$ were 
$\mathrm{HbA} 1 \mathrm{C}<7 \%$

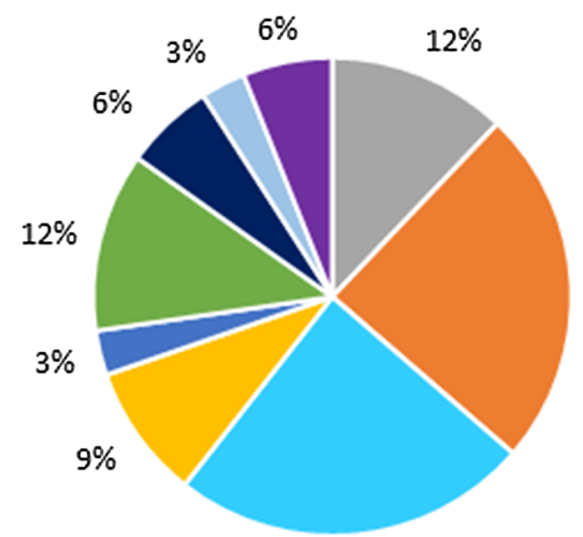

$24 \%$
$25 \%$

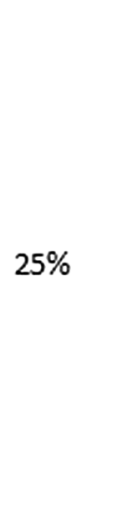

$\mathrm{HbA1C}>7 \%$

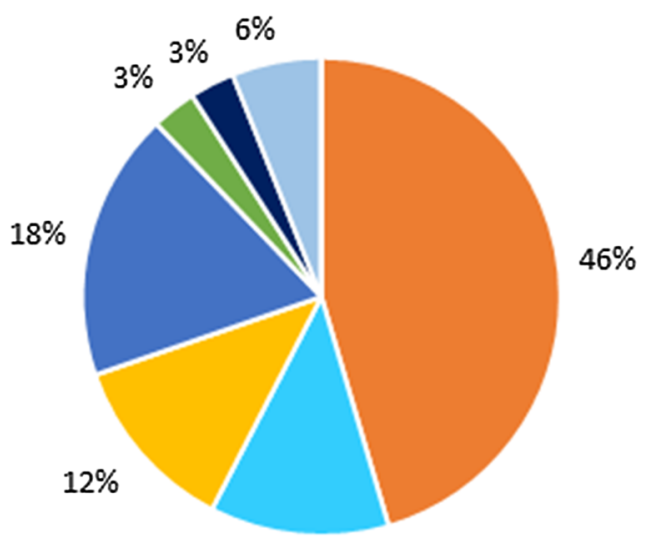

$12 \%$

\begin{tabular}{|c|c|c|c|}
\hline & All Diabetics & $\begin{array}{l}\mathrm{HbA} 1 \mathrm{C}<7 \% \\
\mathrm{~N}=32\end{array}$ & $\begin{array}{l}\mathrm{HbA1C}>7 \% \\
\mathrm{~N}=33\end{array}$ \\
\hline Lifestyle & $5(7 \%)$ & $4(13 \%)$ & $0(0 \%)$ \\
\hline Monotherapy & $45(64 \%)$ & $19(60 \%)$ & $23(70 \%)$ \\
\hline Insulin & 23 & 8 & 15 \\
\hline Metformin & 14 & 8 & 4 \\
\hline Sulfonylurea & 8 & 3 & 4 \\
\hline Bitherapy & $18(26 \%)$ & $7(22 \%)$ & $10(30 \%)$ \\
\hline Insulin+Metformin & 7 & 1 & 6 \\
\hline Sulfonylurea+Gliptines & 5 & 4 & 1 \\
\hline Metformin + Sulfonylurea & 3 & 2 & 1 \\
\hline Metformin + Gliptines & 3 & 1 & 2 \\
\hline $\begin{array}{l}\text { Tritherapy } \\
\quad \text { Insulin + Metformin + Sulfonylurea }\end{array}$ & $2(3 \%)$ & $2(6 \%)$ & $0(0 \%)$ \\
\hline
\end{tabular}

Fig. 3 Hypoglycemic treatment of diabetic patients

more at risk for adverse events (hospitalization for heart failure and all-cause mortality). Previous studies [11, 12, $36,37]$ showed a U-shaped association between HbA1C and prognosis in heart failure patients, with the lowest risk in the group of patients with $\mathrm{HbA} 1 \mathrm{C}$ between 6.5 and $7.5 \%$. However, those studies were either conducted among patients with HFrEF alone, or did not make a distinction between patients according to ejection fraction, while the interplay between diabetes and outcome seems to differ in those populations. In the CHARM trial, the relative risk conferred by diabetes was significantly greater in patients with preserved ejection fraction (EF) than in those with low EF [8] and a recent study highlighted that the presence of T2D was associated with a reduction of exercise capacity (lower peak VO2) in the
LVEF $<40 \%$ and LVEF 40-49\%, but not in the LVEF $>50 \%$ subgroup [38].

Data about glycemic control and outcome in HFpEF are scarce. A study by Gu et al. [13] did not find baseline $\mathrm{HbA} 1 \mathrm{C}$ to be an independent predictor of outcome, but they analyzed it in the overall population of HF with T2D, and not only in HFpEF. Glycemic variability, however, was associated with outcome in the HFpEF subgroup [13] and was associated with signs of diastolic dysfunction in patients without HF [14]. Finally, the GAMIC cohort, a large population-based propensity-matched study of patients with HF [29] observed an increased mortality and morbidity (hospitalizations and visits) in patients who developed diabetes, particularly in those with a mean HbA1c higher than 7.0\%. 


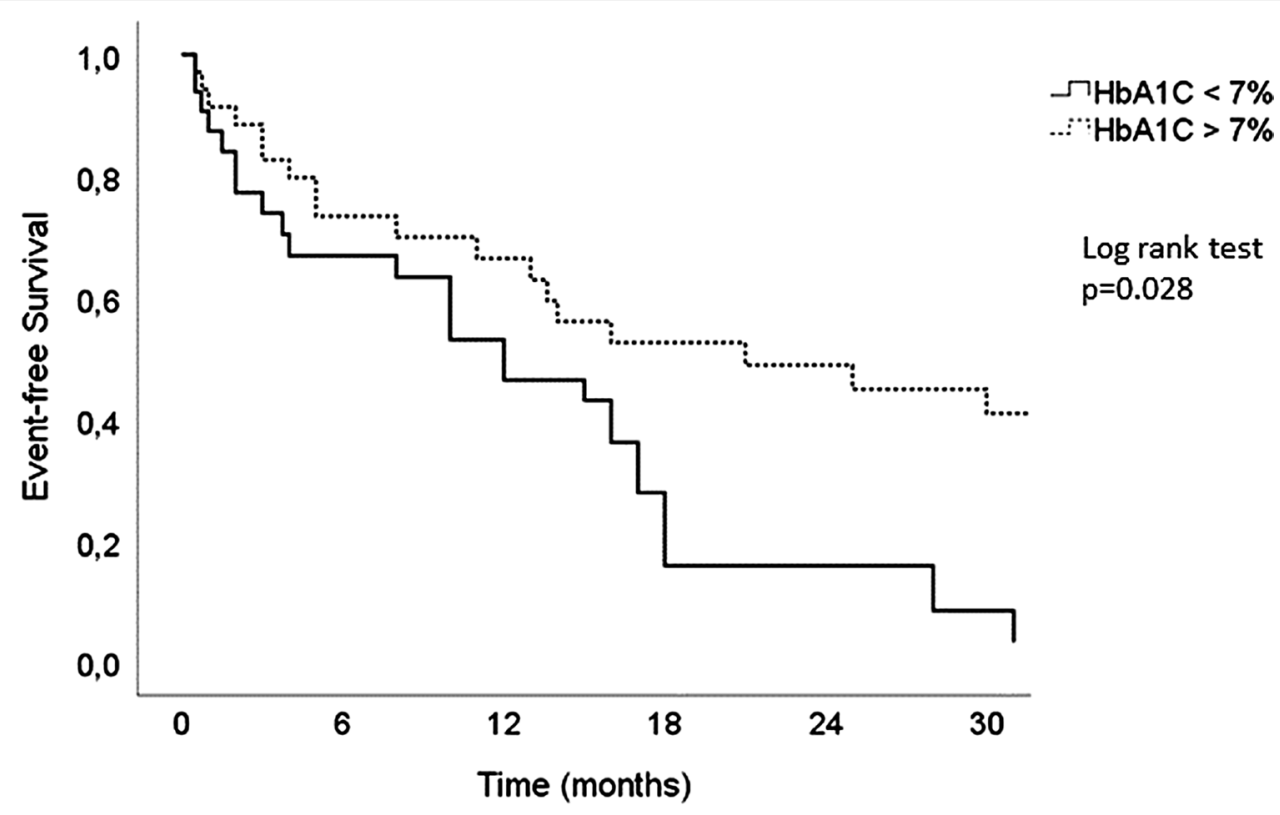

At risk:

$\begin{array}{lllllll}\mathrm{HbA} 1 \mathrm{C}>7 \% & 31 & 22 & 20 & 15 & 13 & 11 \\ \mathrm{HbA} 1 \mathrm{C}<7 \% & 31 & 21 & 16 & 7 & 7 & 5\end{array}$

Fig. 4 Kaplan Meier curves of event-free survival in diabetic HFpEF patients according to HbA1C levels. Adjustments were made for age, body mass index, hemoglobin and NTproBNP levels

How can we explain that, in our population, patients with higher HbA1C levels seem "protected" and suffer from less adverse events, while recent research emphasized the direct role of glucotoxicity on cardiomyocytes in the development of diabetic cardiomyopathy [31, 39, 40]? Firstly, glucotoxicity plays a part in the pathophysiology of the disease but its role in the evolution of symptoms and outcomes is yet unknown. Heart failure in diabetic patients occurs in a broad context of metabolic disorders including lipotoxicity, glucotoxicity and insulin resistance and resulting in impaired mitochondrial oxidative capacity and increased reactive oxygen species (ROS) production and surely, hyperglycemia is not the only mechanism involved. This is supported by the fact that, before the SGLT-2 inhibitor era, no study could demonstrate a favorable effect of glucose lowering therapies on events related to heart failure [41]. Conversely, some glucose-lowering therapies, including peroxisome proliferator-activated receptor (PPAR) agonists even increased the risk of heart failure in individuals with type 2 diabetes. Note that those drugs were seldom taken by patients in our cohort (Fig. 3) and cannot solely be responsible for the difference in event-free survival.

For years, it has been assumed that insulin resistance observed in diseases characterized by nutrient excess (ie T2D and obesity), was fundamental to the pathogenesis of these diseases. As stated above, insulin resistance into the heart has been considered to favor myocardial contractile dysfunction and to be involved in the pathophysiology of diabetic cardiomyopathy. However, an alternative view, which recently gained researchers' interest, is that adaptations occurring in metabolic diseases can be viewed as protective in nature, and that insulin resistance could act as a defense mechanism to prevent or delay pathological intracellular substrate accumulation when substrate uptake exceeds energy demand [42-45]. Fundamental to this hypothesis is that, although these metabolic alterations are deleterious in the long term for complications associated with obesity and diabetes, they provide immediate protection against cell death in response to excess nutrients. Supporting this, it has been shown that cardiac contractile function was preserved, or even improved, in hearts subjected to metabolic and haemodynamic stress when myocardial insulin resistance was induced in response to elevated glucose levels or upon high-fat diet $[46,47]$. Conversely, excessive insulin signaling exacerbates systolic dysfunction when the heart is subjected to pressure overload [48]. In light of this, the discrepancy between our study and the results of the GAMIC cohort [29] might be explained by 
the difference in disease duration. The GAMIC cohort excluded patients with a previous diagnosis of diabetes, while the mean duration of diabetes in our population was $19 \pm 8$ years. Possibly our results do not apply to new onset diabetes, as the adaptation to excess nutrients have not yet taken place.

In this context of old patients with long standing diabetes, the utility of therapeutically targeting glycemia, particularly through insulin sensitization, is questionable as it may result in exposure of cells and tissues to additional nutrients that will further challenge their survival. This could explain why PPAR agonists, important insulin sensitizers favouring nutrient uptake and storage, have been associated with adverse cardiovascular outcomes in T2D patients. On the other hand, treatment reducing nutrient overload might be beneficial and should be preferred. Metformin, for example, which has shown beneficial effect on mortality in HF patients [49], although often referred to as insulin sensitizer, has its main glucoselowering effect via reducing hepatic glucose production. Similarly, SGLT-2 inhibitors lower blood glucose by promoting glycosuria.

This cardioprotective effect of insulin resistance could be involved in the better prognosis observed in heart failure patients with higher BMIs, referred to as the "obesity paradox" $[15,50]$. Although we did not measure insulin resistance per se, we can hypothesize that the group with $\mathrm{HbA} 1 \mathrm{C}>7 \%$ is more insulin resistant as they are more obese and show higher glycemia levels though intensively treated.

Furthermore, hyperglycemia was shown to be involved in irreversible epigenetic changes, known as "glycemic memory", and HbA1c at time of the study cannot reflect the whole history of diabetes [51, 52]. Similarly, intermittent hyperglycemia, rather than chronic elevation of blood glucose, with a lesser repercussion on HbA1C levels, exacerbates the production of reactive oxygen species, impairs endothelial function and induces cytokines release and contributes to pejorative evolution [53]. Finally, hypoglycemia could also be involved in the progression of cardiovascular diseases and mortality through sympatho-adrenal response [54].

In short, together with existing literature, this study underlines that other mechanisms besides glucotoxicity must be involved in the development and worsening of heart failure in diabetic patients, and that the effect of intensive glycemic control on cardiovascular associated morbidity is not fully understood.

Current guidelines recommend that the appropriate target for HbA1C should be individualized based on overall health and life expectancy. As such it is generally accepted that the glycemic goal should be somewhat higher $(\mathrm{HbA} 1 \mathrm{C} \leq 8 \%)$ in frail older adults with medical and functional comorbidities $[55,56]$. Patients with
HFpEF generally match this description (mean age of 78 years and high comorbidity burden in our population). However, these recommendations are based on consensus and there are virtually no trials that have examined glycemic control and complications focusing on the older patients, and even less on older patients with HFpEF. Hence, an important issue that is still unsolved is the optimal target level of HbA1c in that population. Given published data, glycemic variability should be avoided once the optimal target is reached [13]. Our study is a retrospective analysis of a relatively small population and does not allow answering this question. Furthermore, very few patients in our population had severely uncontrolled diabetes. However, this study generates the hypothesis that low levels of $\mathrm{HbA1C}$ are associated with more adverse events, and that physicians should not be too stringent about glycemic control in HFpEF patients with long standing diabetes. In addition, it underlines the need for future studies: fundamental studies to unravel the interaction between diabetes, insulin resistance and heart failure, and clinical studies designed to determine the optimal HbA1C target in HFpEF.

\section{Limitation}

This study was conducted in a single center with a relatively small number of patients. Although data were collected prospectively, the association between HbA1C and mortality were derived from retrospective analyses. As such, this observation is subject to collider stratification bias and our data do not allow generalizing this finding beyond HFpEF patients. The diagnosis of diabetes was reported by investigators and did not require systematic documentation using standardized diagnostic criteria. Its prevalence is, therefore, likely to have been underestimated. Also, unmeasured confounders, such as biomarkers of nutritional status, invasive hemodynamics, and duration of heart disease, that may have improved risk adjustment, were unavailable.

\section{Conclusion}

Together with previous data, this study suggests a potential differentiation of HFpEF phenotypes, with young obese and diabetic HFpEF on one hand, versus elderly HFpEF with atrial fibrillation on the other. This might reflect distinct pathophysiological pathways that perhaps should be targeted more specifically in future clinical trials. Furthermore, these results strengthen evidence on the prognostic significance of diabetes in HFpEF. It underlines that patients with HFpEF and diabetes are at high risk of hospitalization for HF and should benefit of closer monitoring and intensive treatment of comorbidities and congestion. Finally, it shows that a stringent glycemic control has a negative impact on prognosis. This opens the way for future 
research to better understand the interplay between diabetes and heart failure, and to determine an optimal HbA1C target in this specific population.

\section{Supplementary Information}

The online version contains supplementary material available at https://doi. org/10.1186/s12933-021-01242-5.

Additional file 1: Figure S1. Flowchart of the study population. In blue, patients included in descriptive statistics (Tables 1 and 2). In red, patients with complete follow up, used for survival analysis. HbA1C: glycated hemoglobin.

\section{Abbreviations}

AF: Atrial fibrillation; BMI: Body mass index; CMR: Cardiac magnetic resonance; ECV: Extracellular volume; EF: Ejection fraction; eGFR: Estimated glomerular filtration rate; HbA1C: Glycated hemoglobin; HFpEF: Heart failure with preserved ejection fraction; LA: Left atrium; LGE: Late gadolinium enhancement; LV: Left ventricle; NYHA: New York Heart Association; PPAR: Peroxisome proliferator-activated receptor; ROS: Reactive oxygen species; SD: Standard deviation; SGLT-2: Sodium-glucose cotransporter-2; T2D: Type 2 diabetes; TTE: Transthoracic echocardiography.

\section{Acknowledgements}

Figure 1 was adapted from "Servier Medical Art" free online image bank https ://smart.servier.com/smart_image.

\section{Authors' contributions}

SL analyzed and interpreted patients' data and wrote the manuscript. CR and AS prospectively recruited patients and collected data. AP, DV, JLV and BG gave advice on data analyzes and presentation. ACP was the principal investigator of the study. CB and ACP were major contributors in writing the manuscript. All authors read and approved the final manuscript.

\section{Funding}

This work was funded by a grant of the Fondation Nationale de la Recherche Scientifique of the Belgian Government (FRSM CDR 23597851). Dr Pouleur is and Dr Beauloye was supported by a Post-doctorate Clinical Master Specialist of the Fondation Nationale de la Recherche Scientifique of the Belgian Government (FRSM: SPD 10844948). Dr Lejeune is supported by Fondation Damman and Fondation Saint Luc for her fellowship. This work was funded by an unrestricted grant from Astra Zeneca.

\section{Availability of data and materials}

The datasets used and analyzed during the current study are available from the corresponding author on reasonable request.

\section{Ethics approval and consent to participate}

The ethics committee of the Cliniques Universitaire Saint Luc approved the study, and all patients gave written informed consent before study enrolment (Clinical trial NCT03197350). The investigation conforms to the principles outlined in Declaration of Helsinki.

\section{Consent for publication}

Not applicable.

\section{Competing interests}

The authors declare that they have no competing interests.

Received: 23 November 2020 Accepted: 8 February 2021 Published online: 19 February 2021

\section{References}

1. Baral R, Loudon B, Frenneaux MP, Vassiliou VS. Ventricular-vascular coupling in heart failure with preserved ejection fraction: a systematic review and meta-analysis. Heart Lung J Crit Care. 2021;50(1):121-8.

2. Solomon SD, MCMurray JJV, Anand IS, Ge J, Lam CSP, Maggioni AP, et al. Angiotensin-neprilysin inhibition in heart failure with preserved ejection fraction. New Engl J Med. 2019;381(17):1609-20.

3. Nie D, Xiong B, Qian J, Rong S, Yao Y, Huang J. The effect of sacubitrilvalsartan in heart failure patients with mid-range and preserved ejection fraction: a meta-analysis. Heart Lung Circulat. 2020. https://doi. org/10.1016/j.hlc.2020.10.012.

4. Anker SD, Butler J, Filippatos GS, Jamal W, Salsali A, Schnee J, et al. Evaluation of the effects of sodium-glucose co-transporter 2 inhibition with empagliflozin on morbidity and mortality in patients with chronic heart failure and a preserved ejection fraction: rationale for and design of the EMPEROR-Preserved Trial. Eur J Heart Fail. 2019;21(10):1279-87.

5. Mentz RJ, Kelly JP, von Lueder TG, Voors AA, Lam CS, Cowie MR, et al. Noncardiac comorbidities in heart failure with reduced versus preserved ejection fraction. J Am Coll Cardiol. 2014;64(21):2281-93.

6. Lindman BR, Davila-Roman VG, Mann DL, McNulty S, Semigran MJ, Lewis GD, et al. Cardiovascular phenotype in HFpEF patients with or without diabetes: a RELAX trial ancillary study. J Am Coll Cardiol. 2014;64(6):541-9.

7. Kristensen SL, Mogensen UM, Jhund PS, Petrie MC, Preiss D, Win S, et al. Clinical and echocardiographic characteristics and cardiovascular outcomes according to diabetes status in patients with heart failure and preserved ejection fraction: a report from the I-Preserve Trial (Irbesartan in Heart Failure With Preserved Ejection Fraction). Circulation. 2017;135(8):724-35.

8. MacDonald MR, Petrie MC, Varyani F, Ostergren J, Michelson EL, Young JB, et al. Impact of diabetes on outcomes in patients with low and preserved ejection fraction heart failure: an analysis of the Candesartan in Heart failure: assessment of Reduction in Mortality and morbidity (CHARM) programme. Eur Heart J. 2008;29(11):1377-85.

9. Sandesara PB, O'Neal WT, Kelli HM, Samman-Tahhan A, Hammadah M, Quyyumi AA, et al. The prognostic significance of diabetes and microvascular complications in patients with heart failure with preserved ejection fraction. Diabetes Care. 2018;41(1):150-5.

10. Steinberg BA, Zhao X, Heidenreich PA, Peterson ED, Bhatt DL, Cannon $C P$, et al. Trends in patients hospitalized with heart failure and preserved left ventricular ejection fraction: prevalence, therapies, and outcomes. Circulation. 2012;126(1):65-75.

11. Aguilar D, Bozkurt B, Ramasubbu K, Deswal A. Relationship of hemoglobin A1C and mortality in heart failure patients with diabetes. J Am Coll Cardiol. 2009;54(5):422-8.

12. Elder DH, Singh JS, Levin D, Donnelly LA, Choy AM, George J, et al. Mean $\mathrm{HbA} 1 \mathrm{C}$ and mortality in diabetic individuals with heart failure: a population cohort study. Eur J Heart Fail. 2016;18(1):94-102.

13. Gu J, Pan JA, Fan YQ, Zhang HL, Zhang JF, Wang CQ. Prognostic impact of $\mathrm{HbA} 1 \mathrm{c}$ variability on long-term outcomes in patients with heart failure and type 2 diabetes mellitus. Cardiovascular diabetology. 2018;17(1):96.

14. Yokota S, Tanaka H, Mochizuki Y, Soga F, Yamashita K, Tanaka Y, et al. Association of glycemic variability with left ventricular diastolic function in type 2 diabetes mellitus. Cardiovasc Diabetol. 2019;18(1):166.

15. Lejeune S, Roy C, Slimani A, Pasquet A, Vancraeynest D, Beauloye C, et al. Heart failure with preserved ejection fraction in Belgium: characteristics and outcome of a real-life cohort. Acta Cardiol. 2020:1-10.

16. Nagueh SF, Smiseth OA, Appleton CP, Byrd BF 3rd, Dokainish H, Edvardsen $T$, et al. Recommendations for the evaluation of left ventricular diastolic function by echocardiography: an update from the american society of echocardiography and the European association of cardiovascular imaging. Eur Heart J Cardiovas Imag. 2016;17(12):1321-60.

17. Roy C, Slimani A, de Meester C, Amzulescu M, Pasquet A, Vancraeynest D, et al. Associations and prognostic significance of diffuse myocardial fibrosis by cardiovascular magnetic resonance in heart failure with preserved ejection fraction. J Cardiovas Magn Reso. 2018;20(1):55.

18. Kellman P, Wilson JR, Xue H, Ugander M, Arai AE. Extracellular volume fraction mapping in the myocardium, part 1: evaluation of an automated method. J Cardiovas Magn Reson. 2012;14:63.

19. Tromp J, Shen L, Jhund PS, Anand IS, Carson PE, Desai AS, et al. Agerelated characteristics and outcomes of patients with heart failure with preserved ejection fraction. J Am Coll Cardiol. 2019;74(5):601-12. 
20. McHugh K, DeVore AD, Wu J, Matsouaka RA, Fonarow GC, Heidenreich PA, et al. heart failure with preserved ejection fraction and diabetes: JACC State-of-the-Art review. J Am Coll Cardiol. 2019;73(5):602-11.

21. Chirinos JA, Bhattacharya P, Kumar A, Proto E, Konda P, Segers P, et al. Impact of diabetes mellitus on ventricular structure, arterial stiffness, and pulsatile hemodynamics in heart failure with preserved ejection fraction. J Am Heart Assoc. 2019;8(4):e011457.

22. Yap J, Tay WT, Teng TK, Anand I, Richards AM, Ling LH, et al. Association of diabetes mellitus on cardiac remodeling, quality of life, and clinical outcomes in heart failure with reduced and preserved ejection fraction. J Am Heart Assoc. 2019;8(17):e013114.

23. Shen L, Rørth R, Cosmi D, Kristensen SL, Petrie MC, Cosmi F, et al. Insulin treatment and clinical outcomes in patients with diabetes and heart failure with preserved ejection fraction. Eur J Heart Fail. 2019;21(8):974-84.

24. Tan ESJ, Tay WT, Teng TK, Sim D, Leong KTG, Yeo PSD, et al. Ethnic differences in atrial fibrillation in patients with heart failure from Asia-Pacific. Heart. 2019;105(11):842-7.

25. Echouffo-Tcheugui JB, Xu H, DeVore AD, Schulte PJ, Butler J, Yancy CW, et al. Temporal trends and factors associated with diabetes mellitus among patients hospitalized with heart failure: Findings from Get With The Guidelines-Heart Failure registry. Am Heart J. 2016;182:9-20.

26. Kotecha D, Lam CS, Van Veldhuisen DJ, Van Gelder IC, Voors AA, Rienstra M. Heart failure with preserved ejection fraction and atrial fibrillation: vicious twins. J Am Coll Cardiol. 2016;68(20):2217-28.

27. Packer M, Lam CSP, Lund LH, Redfield MM. Interdependence of atrial fibrillation and heart failure with a preserved ejection fraction reflects a common underlying atrial and ventricular myopathy. Circulation. 2020;141(1):4-6.

28. Liu G, Long M, Hu X, Hu CH, Du ZM. Meta-analysis of atrial fibrillation and outcomes in patients with heart failure and preserved ejection fraction. Heart Lung Circu. 2020. https://doi.org/10.1016/j.hlc.2020.10.010.

29. Romero SP, Garcia-Egido A, Escobar MA, Andrey JL, Corzo R, Perez V, et al. Impact of new-onset diabetes mellitus and glycemic control on the prognosis of heart failure patients: a propensity-matched study in the community. Int J Cardiol. 2013;167(4):1206-16.

30. Bidasee KR, Zhang Y, Shao CH, Wang M, Patel KP, Dincer UD, et al. Diabetes increases formation of advanced glycation end products on Sarco(endo) plasmic reticulum Ca2+-ATPase. Diabetes. 2004;53(2):463-73.

31. Ritchie RH, Abel ED. Basic Mechanisms of Diabetic Heart Disease. Circ Res. 2020;126(11):1501-25.

32. Butler J, Hamo CE, Filippatos G, Pocock SJ, Bernstein RA, Brueckmann $\mathrm{M}$, et al. The potential role and rationale for treatment of heart failure with sodium-glucose co-transporter 2 inhibitors. Eur J Heart Fail. 2017;19(11):1390-400.

33. Pollock CA, Lawrence JR, Field MJ. Tubular sodium handling and tubuloglomerular feedback in experimental diabetes mellitus. Am J Physiol. 1991;260(6 Pt 2):F946-52.

34. Zinman B, Wanner C, Lachin JM, Fitchett D, Bluhmki E, Hantel S, et al. Empagliflozin, cardiovascular outcomes, and mortality in type 2 diabetes. New England J Med. 2015;373(22):2117-28.

35. Hwang IC, Cho GY, Yoon YE, Park JJ, Park JB, Lee SP, et al. Different effects of SGLT2 inhibitors according to the presence and types of heart failure in type 2 diabetic patients. Cardiovasc Diabetol. 2020;19(1):69.

36. Lawson CA, Jones PW, Teece L, Dunbar SB, Seferovic PM, Khunti K, et al. Association between type 2 diabetes and all-cause hospitalization and mortality in the UK general heart failure population: stratification by diabetic glycemic control and medication intensification. JACC Heart Fail. 2018;6(1):18-26.

37. McAlister FA, Zheng Y, Westerhout CM, Buse JB, Standl E, McGuire DK, et al. Association between glycated haemoglobin levels and cardiovascular outcomes in patients with type 2 diabetes and cardiovascular disease: a secondary analysis of the TECOS randomized clinical trial. Eur J Heart Fail. 2020;22:2026-34.

38. Abe T, Yokota T, Fukushima A, Kakutani N, Katayama T, Shirakawa R, et al. Type 2 diabetes is an independent predictor of lowered peak aerobic capacity in heart failure patients with non-reduced or reduced left ventricular ejection fraction. Cardiovasc Diabetol. 2020;19(1):142.
39. Jia G, Whaley-Connell A, Sowers JR. Diabetic cardiomyopathy: a hyperglycaemia- and insulin-resistance-induced heart disease. Diabetologia. 2018;61(1):21-8.

40. Battault S, Renguet E, Van Steenbergen A, Horman S, Beauloye C, Bertrand L. Myocardial glucotoxicity: Mechanisms and potential therapeutic targets. Archives of cardiovascular diseases. 2020.

41. Udell JA, Cavender MA, Bhatt DL, Chatterjee S, Farkouh ME, Scirica BM. Glucose-lowering drugs or strategies and cardiovascular outcomes in patients with or at risk for type 2 diabetes: a meta-analysis of randomised controlled trials. Lancet Diabet Endocrinol. 2015;3(5):356-66.

42. Connor T, Martin SD, Howlett KF, MCGee SL. Metabolic remodelling in obesity and type 2 diabetes: pathological or protective mechanisms in response to nutrient excess? Clin Exp Pharmacol Physiol. 2015:42(1):109-15.

43. Taegtmeyer H, Beauloye C, Harmancey R, Hue L. Insulin resistance protects the heart from fuel overload in dysregulated metabolic states. Am J Physiol Heart Circulat Physiol. 2013;305(12):H1693-7.

44. Nolan CJ, Ruderman NB, Kahn SE, Pedersen O, Prentki M. Insulin resistance as a physiological defense against metabolic stress: implications for the management of subsets of type 2 diabetes. Diabetes. 2015;64(3):673-86.

45. Shimizu M, Kario K. Role of the augmentation index in hypertension. Therapeutic Adv Cardiovasc Dis. 2008;2(1):25-35.

46. Harmancey R, Lam TN, Lubrano GM, Guthrie PH, Vela D, Taegtmeyer $H$. Insulin resistance improves metabolic and contractile efficiency in stressed rat heart. FASEB J. 2012;26(8):3118-26.

47. Christopher BA, Huang HM, Berthiaume JM, McElfresh TA, Chen X, Croniger CM, et al. Myocardial insulin resistance induced by high fat feeding in heart failure is associated with preserved contractile function. Am J Physiol Heart Circulat Physiol. 2010;299(6):H1917-27.

48. Shimizu I, Minamino T, Toko H, Okada S, Ikeda H, Yasuda N, et al. Excessive cardiac insulin signaling exacerbates systolic dysfunction induced by pressure overload in rodents. J Clin Investig. 2010;120(5):1506-14.

49. Halabi A, Sen J, Huynh Q, Marwick TH. Metformin treatment in heart failure with preserved ejection fraction: a systematic review and metaregression analysis. Cardiovas Diabetol. 2020;19(1):124.

50. Kapoor JR, Heidenreich PA. Obesity and survival in patients with heart failure and preserved systolic function: a U-shaped relationship. Am Heart J. 2010;159(1):75-80.

51. Pirola L, Balcerczyk A, Tothill RW, Haviv I, Kaspi A, Lunke S, et al. Genomewide analysis distinguishes hyperglycemia regulated epigenetic signatures of primary vascular cells. Genome Res. 2011;21(10):1601-15.

52. Cencioni C, Spallotta F, Greco S, Martelli F, Zeiher AM, Gaetano C. Epigenetic mechanisms of hyperglycemic memory. Int J Biochem Cell Biol. 2014;51:155-8.

53. Monnier L, Mas E, Ginet C, Michel F, Villon L, Cristol JP, et al. Activation of oxidative stress by acute glucose fluctuations compared with sustained chronic hyperglycemia in patients with type 2 diabetes. JAMA. 2006;295(14):1681-7.

54. Skyler JS, Bergenstal R, Bonow RO, Buse J, Deedwania P, Gale EA, et al. Intensive glycemic control and the prevention of cardiovascular events: implications of the ACCORD, ADVANCE, and VA Diabetes Trials: a position statement of the American Diabetes Association and a Scientific Statement of the American College of Cardiology Foundation and the American Heart Association. J Am Coll Cardiol. 2009;53(3):298-304.

55. Kirkman MS, Briscoe VJ, Clark N, Florez H, Haas LB, Halter JB, et al. Diabetes in older adults. Diabetes Care. 2012:35(12):2650-64.

56. Moghissi E. Management of type 2 diabetes mellitus in older patients: current and emerging treatment options. Diabetes Therapy. 2013;4(2):239-56.

\section{Publisher's Note}

Springer Nature remains neutral with regard to jurisdictional claims in published maps and institutional affiliations. 